\title{
Aceptabilidad de un tratamiento vía internet para la agorafobia por pacientes y terapeutas
}

\author{
María José Roca-Sánchez \\ Instituto de Biotecnología. Universidad de Granada \\ Yolanda Álvarez-Pérez y Wenceslao Peñate Castro \\ Dpto. de Psicología Clínica, Psicobiología y Metodología. Universidad de La Laguna
}

RESUMEN

Los programas de tratamiento psicológico vía internet han tenido un gran desarrollo. Estos programas varían tanto en sus contenidos como en sus aspectos formales (textos, videos, avatares...), por lo que su aceptabilidad (facilidad de uso, aprendizaje, atractivo...) no está garantizada $y$, sin embargo, la aceptabilidad no suele evaluarse. El objetivo de este trabajo es la evaluación de la aceptabilidad, por 15 terapeutas y 14 pacientes, de un programa cognitivo-conductual vía internet para el tratamiento de la agorafobia (doctoragora. com/demo). Se elaboró una escala de 10 ítems que estuvo formada por dos componentes de aceptabilidad (positivo y negativo). En general, tanto terapeutas como pacientes tienen una buena aceptación del programa, aunque los pacientes señalan una cierta complejidad en su manejo y la necesidad de mayor aprendizaje. Los terapeutas indicaron que el programa era muy extenso. Estos resultados se discuten de acuerdo con la necesidad de evaluar la aceptabilidad de cualquier recurso en telesalud.

Palabras clave: aceptabilidad, telesalud, tratamiento vía internet, agorafobia.
ABstract

Internet-based psychological treatments have had a great development. Online therapies are now available for most of disorders and psychological problems. These programs can vary not only in their contents, but also in its formal aspects (texts, videos, avatars ...), so that its acceptability (usability, learning, attractive ...) is not guaranteed and yet, acceptability is rarely assessed. The aim of this paper was to assess, by 15 therapists and 14 patients, a cognitive-behavioral internet-based treatment for agoraphobia (doctoragora.com/demo). A 10-item scale was developed and was formed by two components of acceptability (positive and negative). In general, both therapists and patients have a good acceptance of the program, although patients stated difficulties due to their complexity, and the need for greater learning. Therapists indicate that the program is very extensive. These results are discussed according to the need to assess acceptability prior to using any resource in telehealth.

Keywords: acceptability, telehealth, internetbased treatment, agoraphobia. 


\section{Introducción}

El uso de recursos tecnológicos en la evaluación y tratamiento psicológico ha tenido un gran incremento en los últimos años (Peñate, 2012; Peñate, Roca-Sánchez y del Pino-Sedeño, 2014). Dentro de estos recursos, la telesalud (servicios y recursos computarizados en relación con la salud que se prestan usualmente vía internet), ha sido uno de los recursos con mayor crecimiento. Dentro de la telesalud, los programas de tratamiento psicológico vía internet (TPI) han destacado especialmente. Este crecimiento se ha debido, sobre todo, a las numerosas barreras que existen para proporcionar un tratamiento psicológico de calidad a las personas que sufren trastornos mentales, ya sea por problemas geográficos o de transporte, económicos o problemas de tiempo disponible (Yuena, 2003).

Los TPIs representan una nueva forma más rápida y flexible de proporcionar tratamientos autoadministrados o tratamientos basados en manuales, cuya eficacia ha sido comprobada empíricamente (Wilson, 1996, 1997). En términos generales, son protocolos estructurados de tratamiento que incluyen psicoeducación tanto sobre el trastorno en sí mismo como de la evaluación psicológica, los componentes de la terapia y la secuencia del tratamiento. Las revisiones sistemáticas y meta-análisis (i.e., Andrews et al., 2010; Baumeister, Reichler, Munzinger y Lin, 2014; Newman, Szkodny, Llera y Przeworski, 2011; Richards y Richardson, 2012) permiten obtener algunas conclusiones sobre la eficacia de estos procedimientos: los TPIs son más eficaces en comparación con un grupo no tratado (o tratado como usualmente) y pueden ser igualmente eficaces que los tratamientos cara a cara; esta eficacia es mayor en problemas con gravedad leve o moderada; los aplicativos más eficaces son los más estructurados, basados en un acercamiento cognitivoconductual; y el contacto (programado o a demanda) con un terapeuta mejora la eficacia.

Los TPIs más frecuentes han sido para el tratamiento de los problemas de ansiedad y fobias (i.e., Newman et al., 2011). Una de las razones más importantes es que los trastornos de ansiedad suelen ser los más prevalentes entre los trastornos mentales (ESEMeD, 2004; Kessler, Petukhova, Sampson, Zaslavsky y Wittchen, 2012). Se disponen de recursos para el tratamiento de problemas de ansiedad como la ansiedad generalizada, pánico, estrés postraumático y para diferentes tipos de fobias. Dentro de estas fobias destaca la agorafobia, ya que está considerada la fobia más grave y la más incapacitante, pues afecta a múltiples dominios de la vida de la persona y, por sus características (miedo a estar en lugares o situaciones de los cuales pueda ser difícil o embarazoso escapar o en los cuales pueda no disponerse de ayuda en el caso de tener un ataque de pánico o síntomas similares a los del pánico), pueden recluir a los pacientes en sus casas. En estos casos, a las ventajas mencionadas de los TPIs, se añade el hecho de que los propios síntomas de la agorafobia pueden impedir el recibir un tratamiento cara a cara, por lo que un tratamiento vía internet puede ser la mejor opción disponible (Peñate, 2012).

Los TPIs también poseen una serie de insuficiencias o limitaciones (Andersson y Titov, 2014), que suelen estar en las dificultades que 
conlleva obtener conclusiones de procedimientos caracterizados por su variedad en un amplio rango de variables (tipo de muestra/ problema, duración, acceso, características formales del aplicativo...). Una de las limitaciones más importantes hace referencia a la adherencia a las TPIs (van Ballegooijen, Cuijpers, van Straten, Karyotaki, y Andersson, 2014), ya que un aspecto central para adherir a los pacientes es la aceptabilidad del recurso web. La aceptabilidad hace referencia al grado en el que los pacientes (u otros usuarios) están satisfechos o cómodos con un servicio y están dispuestos a utilizarlo nuevamente. Este concepto implica aspectos como la confortabilidad, acceso sencillo, el aprendizaje fácil y una presentación atractiva, que hace que el recurso sea fiable y válido (Rush y Scott, 2004). Los indicadores de aceptabilidad incluirían la comprensibilidad, el aprendizaje, la operatividad, la satisfacción y las percepciones de los diferentes grupos interesados (Gun, 2011).

En la actualidad hay una proliferación de la literatura que explora la fiabilidad, eficacia y aceptabilidad de los tratamientos por internet (Antonacci, Bloch, Saeed, Yildirim y Talley, 2008; Bee, 2010; Hommel, 2013; Mitchell, 2008; Wootton, 2011), porque de ello se deriva el que las personas se adhieran al tratamiento $y$, consecuentemente, pueda resultar un tratamiento eficaz. En este sentido, eficacia y aceptabilidad no deben confundirse, ya que, por un lado, puede darse el caso de que un programa sea aceptable (usable, amigable) pero escasamente eficaz, porque sus contenidos no abordan adecuadamente el problema o trastorno en cuestión; y, por otro lado, se pueden encontrar programas basados en tratamientos eficaces pero su acceso y diseño no les hace ser aceptables para los usuarios, por lo que no se van a adherir al programa $y$, consecuentemente, éste no va a ser eficaz en el abordaje del problema a tratar.

Lo que se presenta a continuación es la aceptabilidad de un programa de tratamiento vía internet para el tratamiento de la agorafobia que, como se ha señalado, es la fobia más incapacitante y que, por su propios síntomas, puede impedir que las pacientes puedan recibir un tratamiento directo cara a cara. EI TPI se denomina doctoragora (wwwdoctoragora. com/demo) y está fundamentado en un tratamiento cognitivo conductual bien establecido de 11 sesiones de tratamiento. Combina técnicas de desactivación, reestructuración cognitiva y exposición a estímulos virtuales fóbicos y ha mostrado su eficacia experimental y clínica (Peñate et al., 2014; Pitti et al., 2015).

Así pues, el objetivo de este estudio fue evaluar la aceptabilidad del programa doctoragora por un grupo de pacientes y terapeutas. Secundariamente fue necesario construir una pequeña escala para evaluar dicha aceptabilidad, de acuerdo a los contenidos en relación con la comprensión, aprendizaje, operatividad $y$ atractivo del recurso web.

\section{Método}

\section{Participantes}

Un total de 15 terapeutas, todos ellos con la especialidad de clínica (67\% mujeres), con una edad comprendida entre los 37 y los 58 años y con 10 años o más de experiencia (entre los 10 años de experiencia y los 30, el $20 \%$ tenía 
26 años de práctica clínica) y 14 pacientes (79\% mujeres) con una edad comprendida entre los 25 y los 52 años y, al menos, 5 años de evolución del trastorno compusieron la muestra final.

La captación de pacientes se realizó a través de su derivación desde las diferentes unidades de salud mental de Tenerife, así como por medio de una nota de prensa publicada en el periódico.

Los criterios de inclusión para los pacientes consistieron en: que cumplieran los criterios de la entrevista ICD-10 (World Health Organization, WHO, 1992) para el diagnóstico de agorafobia (con/sin pánico) y 5 años o más de evolución del trastorno. Los criterios de exclusión de los pacientes fueron: síntomas psicóticos o trastorno bipolar, alto riesgo de suicidio y trastornos neurológicos (como epilepsia).

Los criterios de inclusión de los terapeutas consistieron en: estar especializados en clínica, 10 años o más de práctica clínica. No se determinaron criterios de exclusión.

El cuestionario de aceptabilidad fue administrado vía mail a los participantes (tanto pacientes como profesionales). Ningún incentivo fue ofrecido a los participantes.

Descripción de la web doctoragora.com/ demo.

El programa Doctoragora se inició en el 2009 con un proyecto financiado por el Ministerio de Ciencia e Innovación. Dirección General de Programas y Transferencia de Conocimiento, con la referencia: PSI 2009-09836 (subprograma PSIC), en el que participa la Universidad de La Laguna. Doctoragora.com/demo tiene una orientación cognitivo-conductual y está basado en las guías clínicas que se han derivado de los tratamientos eficaces. Cons- ta de 11 sesiones donde la primera sesión es psicoeducativa (qué es la agorafobia, origen, curso, etc.), la segunda y tercera son de reestructuración cognitiva y técnicas de relajación. El resto de las sesiones son una combinación de exposición en vivo y exposición en Realidad Virtual (mediante la descarga de los escenarios virtuales). A lo largo de todas las sesiones, el paciente realiza autorregistros y elabora listas de situaciones temidas que envía al terapeuta vía correo electrónico (Tabla 1).

El acceso al programa doctoragora es sencillo y puede hacerse desde cualquier ordenador con conexión a internet (En la figura 1 se recoge la página de inicio de la web). Su acceso es gratuito y su manejo es por medio de menús interactivos. Está fundamentado en técnicas de exposición aproximativa, es decir, se enseña al paciente a perder sus miedos de una manera progresiva, de forma que el proceso se adapte a sus características personales.

\section{Cuestionario de aceptabilidad}

De acuerdo con la guía propuesta sobre los contenidos de la aceptabilidad (Rush y Scott, 2004), se construyó un cuestionario sencillo de 10 ítems (Anexo 1) con el propósito de evaluar cuatro conceptos: comprensión, aprendizaje, operatividad y satisfacción (atractivo). El formato de la escala fue tipo Likert con 4 opciones de respuesta (muy de acuerdo, de acuerdo, en desacuerdo, muy en desacuerdo) y una pregunta abierta para dar la posibilidad de que tanto los pacientes como los terapeutas opinasen sobre las mejoras que podrían realizarse en la web.

Todos los participantes fueron informados y completaron el consentimiento informado antes de comenzar el programa. 
Tabla 1

Descripción de la Web doctoragora.com/demo.

\begin{tabular}{|c|c|c|}
\hline SESIÓN & CONTENIDO & CUESTIONARIOS/TAREAS \\
\hline $1^{\mathrm{a}}$ & $\begin{array}{l}\text { Psicoeducativa: ¿qué es la agorafobia?, } \\
\text { origen, tratamiento. }\end{array}$ & Cuestionarios \\
\hline $2^{a}$ & Técnicas para disminuir la ansiedad & Descargar audios y textos de relajación \\
\hline $3^{a}$ & $\begin{array}{l}\text { Distorsiones Cognitivas. Reestructuración } \\
\text { cognitiva }\end{array}$ & Autorregistros \\
\hline $4^{a}$ & $\begin{array}{l}\text { Exposición. Identificar situaciones temidas } \\
\text { mediante un protocolo. } \\
\text { Exposición realidad virtual }\end{array}$ & $\begin{array}{l}\text { Autorregistros } \\
\text { Valoración USAS situaciones temidas } \\
\text { Descargar escenarios visuales }\end{array}$ \\
\hline $5^{\mathrm{a}}$ & Exposición en vivo y exposición virtual & $\begin{array}{l}\text { Autorregistros } \\
\text { Valoración USAS situaciones temidas } \\
\text { Escenarios virtuales }\end{array}$ \\
\hline $6^{a}$ & Exposición en vivo y exposición virtual & $\begin{array}{l}\text { Autorregistros } \\
\text { Valoración USAS situaciones temidas } \\
\text { Escenarios virtuales }\end{array}$ \\
\hline $7^{a}$ & $\begin{array}{l}\text { Exposición en vivo y exposición virtual } \\
\text { Conductas de afrontamiento desadaptativas } \\
\text { (CAD): tareas distractorias, supersticiosas, } \\
\text { elementos de seguridad. }\end{array}$ & $\begin{array}{l}\text { Autorregistros } \\
\text { Valoración USAS situaciones temidas } \\
\text { Escenarios virtuales }\end{array}$ \\
\hline $8^{a}$ & $\begin{array}{l}\text { Exposición en vivo y exposición virtual } \\
\text { CAD }\end{array}$ & $\begin{array}{l}\text { Autorregistros } \\
\text { Valoración USAS situaciones temidas } \\
\text { Escenarios virtuales }\end{array}$ \\
\hline $9^{a}$ & $\begin{array}{l}\text { Exposición en vivo y exposición virtual } \\
\text { Enfrentar las } C A D\end{array}$ & $\begin{array}{l}\text { Autorregistros } \\
\text { Valoración USAS situaciones temidas } \\
\text { Escenarios virtuales }\end{array}$ \\
\hline $10^{a}$ & $\begin{array}{l}\text { Exposición en vivo y exposición virtual } \\
\text { Enfrentar las } C A D\end{array}$ & $\begin{array}{l}\text { Autorregistros } \\
\text { Valoración USAS situaciones temidas } \\
\text { Escenarios virtuales }\end{array}$ \\
\hline $11^{\mathrm{a}}$ & $\begin{array}{l}\text { Programación: repaso lugares y situaciones } \\
\text { superadas (vivo). } \\
\text { Repaso de conocimientos y motivación para } \\
\text { continuar practicando: enfrentamiento y } \\
\text { técnicas de relajación. }\end{array}$ & $\begin{array}{l}\text { Autorregistros } \\
\text { Valoración USAS situaciones temidas } \\
\text { Cuestionarios } \\
\text { Visita del terapeuta }\end{array}$ \\
\hline
\end{tabular}

\section{Resultados}

Un primer grupo de análisis se llevó a cabo para conocer las relaciones entre los diferentes ítems de la escala de aceptabilidad. En la Tabla 2 se resumen los coeficientes obtenidos.

Como puede observarse, dos ítems son los que mantienen mayores relaciones significativas con el resto de los ítems. Así, el ítem2 (co- nocimiento para conectarse al programa) se relaciona significativamente con el conocimiento del funcionamiento de la web (ítem3), con la rapidez en aprender a manejar el programa (ítem4), confianza en el manejo de la misma (ítem6) y atractivo de la página (ítem8). Por otro lado, el mencionado ítem6 (confianza), además de las relaciones positivas con el ítem2, covaría 


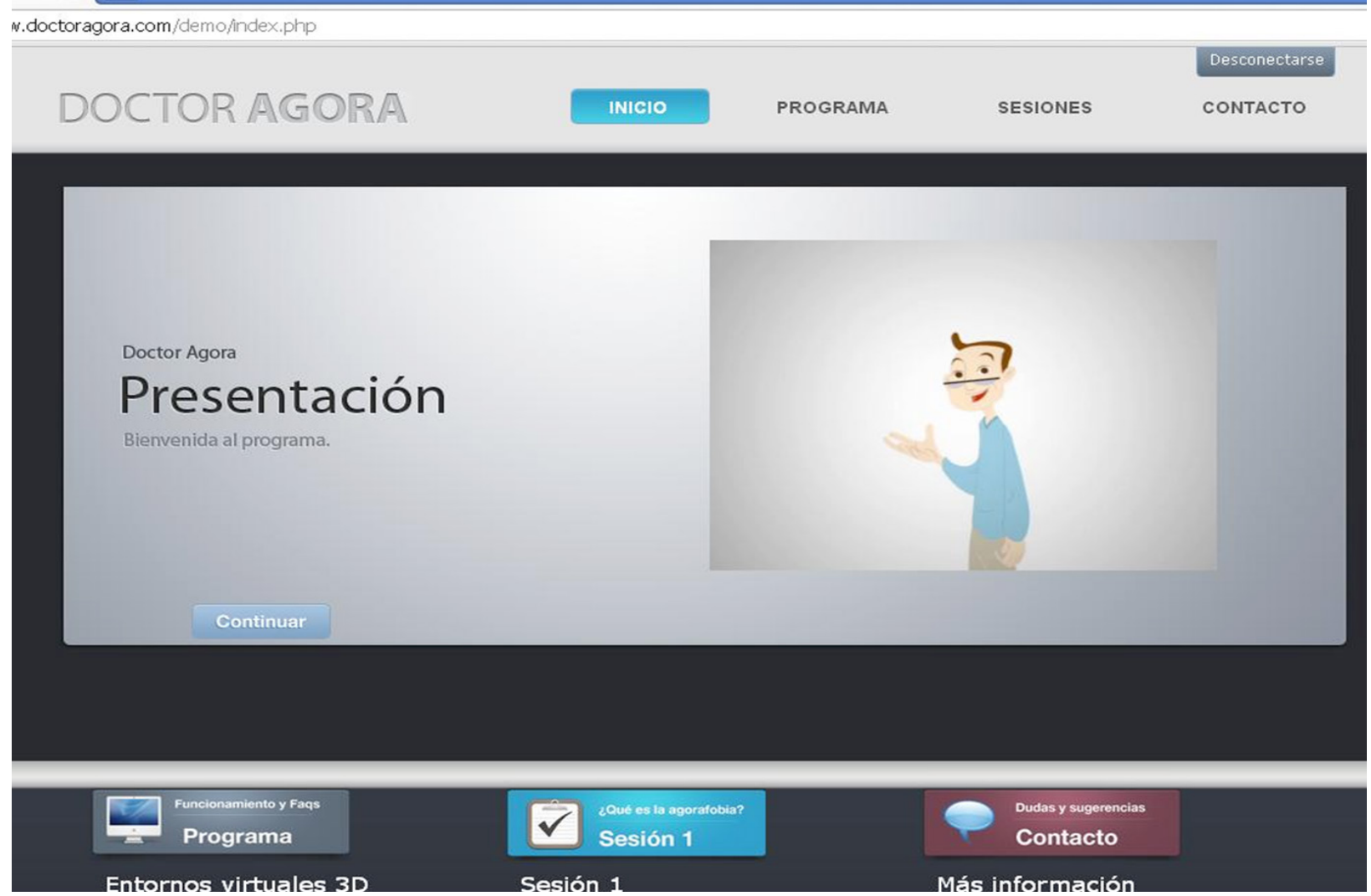

Figura 1. Página de inicio del programa doctoragora.com/demo

también con los mismos ítems: ítem3 (conocimiento de la web), ítem4 (rapidez en aprender) y con el ítem8 (atractivo), conformando una estructura de aceptabilidad entre ellos. También los ítems con contenidos negativos (ítem1, complejidad; ítem5, extensión; ítem7, necesidad de mayor aprendizaje), mantienen relaciones entre sí, dando cuenta de una doble estructura de aceptabilidad (positiva y negativa). Esta doble estructura se muestra en el análisis de componentes que se resume en la Tabla 3.

En este caso se puede observar cómo los ítems de aceptabilidad positiva (conocimiento, facilidad de aprendizaje, confianza y atractivo de la web) conforman un componente; mientras que los negativos (complejidad, extensión y necesidad de mayor aprendizaje) conforman la estructura negativa. En este caso, además (en sentido positivo) se añade el ítem de facilidad de lectura de los textos del programa. El ítem 10 (recomendar la web a otra persona), se mantiene de manera independiente. La consistencia interna del primer componente (aceptabilidad positiva) obtuvo un alfa de cronbach de 0,78 , mientras que el componente de aceptabilidad negativa obtuvo un coeficiente de consistencia de 0,75. La correlación entre ambos componentes fue moderada $\left(r_{x y}=-0,21\right)$.

Visto el comportamiento de la escala, se analizaron la aceptabilidad específica por cada grupo de participantes. En la Tabla 4 se resumen las frecuencias de las respuestas dadas por los terapeutas y por los pacientes. Los dos grupos han tenido una buena aceptación de 
Tabla 2.

Correlaciones inter-ítem de la escala de aceptabilidad (muestra total, $n=29$ ).

\begin{tabular}{|c|c|c|c|c|c|c|c|c|c|}
\hline Ítems & 2 & 3 & 4 & 5 & 6 & 7 & 8 & 9 & 10 \\
\hline 1. Encontré la página web muy compleja &,- 32 &,- 27 &,- 07 &, $78^{* *}$ & ,04 &, $79^{* *}$ & ,29 & ,23 &,- 08 \\
\hline $\begin{array}{l}\text { 2. Sé como conectarme como usuario a } \\
\text { la web }\end{array}$ & &, $40^{*}$ &, $64^{* *}$ &,- 02 & $43^{*}$ & ,35 & $37^{*}$ & 14 & ,04 \\
\hline $\begin{array}{l}\text { 3. Entiendo cómo funciona la web de ma- } \\
\text { nera global }\end{array}$ & & & ,34 &,- 07 & $39^{*}$ &,- 29 & ,33 &, 25 &,- 09 \\
\hline $\begin{array}{l}\text { 4. Considero que la mayoría de las per- } \\
\text { sonas aprenderían muy rápidamente a } \\
\text { utilizar la web }\end{array}$ & & & &,- 08 &, $54^{* *}$ &,- 04 & ,33 & ,21 & ,12 \\
\hline $\begin{array}{l}\text { 5. Encontré la web demasiado extensa al } \\
\text { recorrerla }\end{array}$ & & & & &,- 10 &, $73^{* *}$ &,- 21 &,- 02 &,- 14 \\
\hline $\begin{array}{l}\text { 6. Me sentí muy confiado/a en el manejo } \\
\text { de la web }\end{array}$ & & & & & &,- 01 & $44^{*}$ & 35 & ,27 \\
\hline $\begin{array}{l}\text { 7. Necesito aprender muchas más cosas } \\
\text { antes de manejarme bien en la web }\end{array}$ & & & & & & & ,26 & ,22 &,- 04 \\
\hline 8. Me ha gustado el diseño de la web & & & & & & & &,- 04 & ,13 \\
\hline 9. Me ha resultado fácil leer el contenido & & & & & & & & &, $53^{* *}$ \\
\hline $\begin{array}{l}\text { 10. Recomendaría el uso de la web a per- } \\
\text { sonas que padecieran agorafobia }\end{array}$ & & & & & & & & & \\
\hline
\end{tabular}

Nota: ${ }^{* *}=p \leq .01 ;{ }^{*}=p \leq .05$

la web, mostrando una mayor frecuencia de 'acuerdos' en los ítems positivos. Sin embargo, en los ítems negativos, mientras que los terapeutas muestran su acuerdo con uno de ellos (ítem5, relacionado con su acuerdo con que la web es demasiado extensa), los pacientes expresan su acuerdo en que la web es compleja (ítem1) y que necesitan más aprendizaje (ítem5). Agrupando las respuestas en 'acuerdos' y 'desacuerdos' y contrastando los dos grupos

Tabla 3.

Resumen del análisis de componentes principales sobre la escala de aceptabilidad.

\begin{tabular}{|c|c|c|c|c|}
\hline & & \multicolumn{3}{|c|}{ Saturación componentes } \\
\hline & & 1 & 2 & 3 \\
\hline 1. & Encontré la página web muy compleja & 29 &,- 92 &,- 08 \\
\hline 2. & Sé como conectarme como usuario a la web & 81 &,- 23 & ,08 \\
\hline 3. & Entiendo cómo funciona la web de manera global & ,66 &,- 26 & 07 \\
\hline 4. & $\begin{array}{l}\text { Considero que la mayoría de las personas aprenderían muy } \\
\text { rápidamente a utilizar la web }\end{array}$ & 79 &, 05 & 19 \\
\hline 5. & Encontré la web demasiado extensa al recorrerla & 03 &,- 90 &,- 13 \\
\hline 6. & Me sentí muy confiado/a en el manejo de la web & ,75 & ,08 & ,42 \\
\hline & $\begin{array}{l}\text { Necesito aprender muchas más cosas antes de manejarme } \\
\text { bien en la web }\end{array}$ & 27 &,- 91 & ,06 \\
\hline 8. & Me ha gustado el diseño de la web & ,64 &,- 29 & 02 \\
\hline & Me ha resultado fácil leer el contenido & ,24 & ,32 & ,09 \\
\hline & $\begin{array}{l}\text { Recomendaría el uso de la web a personas que padecieran } \\
\text { agorafobia }\end{array}$ & ,09 & 13 & 86, \\
\hline
\end{tabular}


(chi cuadrado), se encuentran diferencias significativas entre los dos en esos tres ítems: ítem1: $X^{2}(1)=21,93(p=0,001)$; ítem5: $X^{2}(1)=16,35(p$ $=0,000)$; e ítem7: $\left(X^{2}(1)=21,93(p=0,001)\right.$.

Tomando estos ítems de acuerdo a los dos componentes conformados, no se observaron diferencias significativas entre pacientes y terapeutas en la aceptabilidad positiva $[\mathrm{F}(1)=$ $0,53]$, pero sí se encontraron para la aceptabilidad negativa $[F(1)=101,03 ; p=0,000]$, siendo los pacientes los que mostraron mayores niveles en este componente.

En cuanto a la pregunta abierta " $¿ Q u e ́$ cosas cambiaría o mejoraría en la web?", de los 14 pacientes que completaron el cuestionario, sólo 11 contestaron a esta pregunta. Las respuestas se pueden organizar en mejoras a nivel operacional, es decir, de acceso, formato y desarrollo de la página; a nivel motivacional y relacionadas con la descarga y utilización de los escenarios virtuales.(Tabla 5).

Ningún terapeuta contestó a la pregunta.

\section{Discusión}

La proliferación de programas de tratamiento psicológico vía internet ha tenido un gran crecimiento. Este desarrollo ha supuesto una avance importante, ya que se dispone de recursos de fácil acceso para personas que, por diferentes motivos (movilidad restringida, dificultades geográficas, inexistencia de recursos sanitarios, problemas de tiempo...), no pueden acceder a un tratamiento en directo. Sin embargo, este avance no se ha visto acompañado siempre por la evaluación de la aceptabilidad de dichos recursos, presuponiéndose con frecuencia que el programa estaba bien diseñado y los pacientes lo iban a aceptar. Secundariamente, la aceptación del programa se derivaría de su eficacia. Sin embargo, la aceptabilidad (Rush y Scott, 2004) es un concepto relacionado directamente con el diseño del aplicativo informático y en qué medida éste supone una recurso sencillo, amigable, de fácil comprensión/aprendizaje, atractivo. Estos componentes pueden hacer

Tabla 4.

Distribución de las respuestas a los ítems por categorías y por terapeutas $(T)$ y pacientes $(P)$.

\begin{tabular}{|c|c|c|c|c|c|c|c|c|}
\hline \multirow{2}{*}{ Ítems } & \multicolumn{2}{|c|}{$\begin{array}{c}\text { Muy en } \\
\text { desacuerdo }\end{array}$} & \multicolumn{2}{|c|}{$\begin{array}{c}\text { En } \\
\text { desacuerdo }\end{array}$} & \multicolumn{2}{|c|}{$\begin{array}{l}\text { De } \\
\text { acuerdo }\end{array}$} & \multicolumn{2}{|c|}{$\begin{array}{l}\text { Muy de } \\
\text { acuerdo }\end{array}$} \\
\hline & $\mathrm{T}$ & $P$ & $\mathrm{~T}$ & $P$ & $\mathrm{~T}$ & $P$ & $\mathrm{~T}$ & $\mathrm{P}$ \\
\hline 1. Encontré la página web muy compleja & 9 & 1 & 6 & 3 & 0 & 6 & 0 & 4 \\
\hline 2. Sé cómo conectarme como usuario a la web & 0 & 1 & 0 & 0 & 6 & 2 & 9 & 11 \\
\hline 3. Entiendo cómo funciona la web de manera global & 0 & 0 & 1 & 3 & 8 & 4 & 6 & 7 \\
\hline $\begin{array}{l}\text { 4. Considero que la mayoría de las personas apren- } \\
\text { derían muy rápidamente a utilizar la web }\end{array}$ & 0 & 0 & 0 & 2 & 7 & 6 & 8 & 6 \\
\hline 5. Encontré la web demasiado extensa al recorrerla & 0 & 5 & 3 & 7 & 12 & 1 & 0 & 1 \\
\hline 6. Me sentí muy confiado/a en el manejo de la web & 0 & 0 & 0 & 3 & 7 & 7 & 8 & 4 \\
\hline $\begin{array}{l}\text { 7. Necesito aprender muchas más cosas antes de } \\
\text { manejarme bien en la web }\end{array}$ & 8 & 1 & 7 & 1 & 0 & 5 & 0 & 7 \\
\hline 8. Me ha gustado el diseño de la web & 0 & 0 & 5 & 3 & 8 & 9 & 0 & 2 \\
\hline 9. Me ha resultado fácil leer el contenido & 0 & 1 & 0 & 0 & 4 & 4 & 11 & 9 \\
\hline $\begin{array}{l}\text { 10. Recomendaría el uso de la web a personas que } \\
\text { padecieran agorafobia }\end{array}$ & 0 & 0 & 0 & 0 & 2 & 5 & 13 & 9 \\
\hline
\end{tabular}


Tabla 5.

Respuestas de los pacientes a la pregunta abierta sobre mejora de la web

\begin{tabular}{|c|c|c|}
\hline Operativo & Motivacional & Escenarios Virtuales \\
\hline $\begin{array}{l}\text { "Creo que podría facilitar el uso de } \\
\text { la web, la no existencia de tanta } \\
\text { pestaña, sino que el programa te } \\
\text { lleve directamente a la siguiente } \\
\text { parte. Que guarde la información } \\
\text { de lo que has hecho. La existencia } \\
\text { de unos plazos para que se } \\
\text { habiliten las sesiones, aunque soy } \\
\text { consciente de que se ha hecho con } \\
\text { la finalidad de que exista un plazo } \\
\text { para interiorizar el aprendizaje, me } \\
\text { parece un obstáculo". }\end{array}$ & $\begin{array}{l}\text { "Incluiría también un mensaje de } \\
\text { bienvenida de motivación, tipo } \\
\text { frases típicas, siempre diferentes } \\
\text { cada vez que entrara. Ejemplo: "Si } \\
\text { quieres resultados diferentes, no } \\
\text { hagas siempre lo mismo". Quizás, } \\
\text { proponer ejercicios fáciles, en } \\
\text { frases emergentes, tipo sugerencia } \\
\text { motive más. Ejemplo: "Hoy quizás } \\
\text { podrías comprarte una tarta de } \\
\text { chocolate". }\end{array}$ & $\begin{array}{l}\text { "Lo más complicado ha sido el } \\
\text { acceso a los escenarios virtuales". }\end{array}$ \\
\hline $\begin{array}{l}\text { "Pondría los audios y las lecturas } \\
\text { en páginas separadas. Primero la } \\
\text { lectura y después el audio". }\end{array}$ & $\begin{array}{l}\text { "Añadir una pequeña red social, } \\
\text { tipo foro, para los usuarios, que } \\
\text { comenten entre ellos sus avances } \\
\text { (sólo avances, no fracasos) quizás } \\
\text { ayude a los demás a pensar un } \\
\text { poco diferente". }\end{array}$ & $\begin{array}{l}\text { "...el problema que puede dar } \\
\text { el formato de descarga de los } \\
\text { escenarios virtuales...". }\end{array}$ \\
\hline $\begin{array}{l}\text { "Almacenaría el seguimiento del } \\
\text { usuario. Es decir, que se inicie } \\
\text { donde lo dejaste". }\end{array}$ & $\begin{array}{l}\text { "Sería muy fácil, poner un mapa } \\
\text { de Google Maps, en el cual cada } \\
\text { usuario pone una chincheta nueva } \\
\text { donde ha conseguido llegar y } \\
\text { escribir algún comentario en el } \\
\text { mapa". }\end{array}$ & $\begin{array}{l}\text { "Hay que abrir desde fuera los } \\
\text { escenarios virtuales, deberían } \\
\text { poder abrirse desde el mismo } \\
\text { momento que se poneel contador". }\end{array}$ \\
\hline $\begin{array}{l}\text { "La manera de acceder debería ser } \\
\text { menos compleja, no tantos pasos } \\
\text { a seguir, aunque reconozco que } \\
\text { para pasar de una sesión a otra se } \\
\text { me olvidaba como se entraba". }\end{array}$ & $\begin{array}{l}\text { "Mejoraría el personaje que nos va } \\
\text { explicando todo en la página, una } \\
\text { persona real me gustaría más". }\end{array}$ & \\
\hline $\begin{array}{l}\text { "Aumentaría el número de } \\
\text { escenarios virtuales". }\end{array}$ & & \\
\hline $\begin{array}{l}\text { "No cambiaría nada, porque } \\
\text { el enfoque que le han dado es } \\
\text { perfecto. Pero si las personas que } \\
\text { lo hace no tienen unos mínimos } \\
\text { conocimientos de informática le es } \\
\text { imposible realizar este programa". }\end{array}$ & & \\
\hline
\end{tabular}


más aceptables los componentes específicos de un programa de tratamiento vía internet.

La necesidad de evaluar la aceptabilidad de los TPIs está especialmente indicada por su amplia variabilidad (como puede apreciarse en la revisión de Newman et al., 2011, sólo para ansiedad y depresión). Esta variabilidad no sólo se debe al contenido (orientación psicológica, tipo de terapia, número de sesiones, posibilidad de acceso a un terapeuta...), sino por sus aspectos formales (uso de textos, dibujos, videos, avatares...) y su presentación específica.

Debido a esas consideraciones, el objetivo de este estudio fue evaluar la aceptabilidad de un programa de tratamiento diseñado para pacientes diagnosticado con agorafobia, como paso previo a su utilización como recurso terapéutico. A la aceptabilidad de los pacientes se unió la aceptabilidad de los profesionales, en la medida en que estos recursos podrían ser una estrategia de tratamiento más en su quehacer profesional. Para ello fue necesario construir una escala breve que contemplara los parámetros expuestos sobre aceptabilidad.

La escala se conformó en una doble estructura de aceptabilidad (positiva y negativa) que incluía por igual contenidos de comprensión, aprendizaje, operatividad o atractivo. El ítem que evaluaba la posibilidad de recomendar la web a otras personas se mantuvo como un ítem independiente. Estos dos componentes mantienen unas relaciones moderadas $y$, como era esperable, negativas, entre sí. Son estructuras relativamente sólidas, ya que muestran niveles aceptables de consistencia interna.

De los resultados de aceptabilidad se extrae que tanto los pacientes como los terapeutas encuentran la web aceptable y la recomendarían a personas que sufrieran agorafobia. Aun así, se han podido detectar algunas diferencias significativas entre los grupos. Los pacientes encuentran la web más compleja y necesitan aprender más cosas antes de manejarse bien por ella. Estas consideraciones pueden ser entendidas como una dificultad añadida para el manejo inicial del programa de tratamiento, pero, considerando la opinión de los pacientes sobre el resto de contenidos de la escala (facilidad de acceso, navegación, lectura...), también puede entenderse como la preocupación por los pacientes por saber aprovechar lo mejor posible el recurso web, señalando su preocupación por ser capaces de beneficiarse del programa. En todo caso, estas dos consideraciones conllevaría que los usuarios del doctoragora.com/demo necesitarían de un apoyo adicional por parte de un técnico/terapeuta externo que pueda resolver los problemas planteados por esa complejidad y esa dificultad de aprendizaje. Este contacto parece ser un elemento clave de la eficacia de los tratamientos vía internet (Newman et al., 2011), y que forma parte de los intercambios verbales entre pacientes y clínicos, que son, a su vez, elementos claves del éxito terapéutico (FrojánParga, Galván-Domínguez, Izquierdo-Alfaro, Ruiz-Sancho y Marchena-Giráldez, 2013).

En el caso de los profesionales de la psicología, éstos la encuentran demasiado extensa. Son 11 sesiones, con varios recursos disponibles que deben ser usados (técnicas de relajación, escenarios virtuales...), un proceso de evaluación inicial y el compromiso de enviar autorregistros frecuentes. Se entiende que es- 
tos cometidos escapan al interés de los profesionales $y$, en ese sentido, pueden considerar la web como extensa. Sin embargo, para los pacientes, que van a ser los usuarios prioritarios, esta extensión excesiva no aparece, por lo que es posible que la opinión de los terapeutas se deba más a su consideración personal a la hora de evaluar la aceptabilidad de del aplicativo (muy extenso en relación con contestar una escala breve), que a su utilidad. En este sentido, el hecho de que recomienden la web, puede mediatizar el hecho de que la consideren demasiado extensa para el tratamiento de la agorafobia.

Si nos centramos en la pregunta abierta, se puede considerar que esto que se menciona se debe sobre todo a la descarga de los escenarios virtuales y a las características de diseño de la web: plazos de habilitación de las sesiones (dos días mínimo), la existencia de pestañas para pasar de sesión a sesión, etc., y a características intrínsecas de los usuarios como puede ser su nivel de alfabetización informática.

En definitiva, la aceptabilidad del programa puede considerarse apropiada, entendiendo que se necesita de un apoyo técnico y profesional específico en relación con la complejidad del mismo para algunos pacientes y su necesidad de algún apoyo adicional para un mejor aprendizaje de las estrategias que se proponen.

Este es un estudio preliminar con claras limitaciones: una muestra pequeña, una escala nueva que necesitará mayor validación y unos contenidos que habría que complementar con otros más en relación con la usabilidad, amigabilidad y aceptabilidad del recurso web. Sin embargo, se considera que, a pesar de estas limitaciones, la evaluación de la aceptabilidad de un tratamiento psicológico que se presta vía internet se hace necesaria antes de su aplicación como recurso terapéutico y no debe confundirse con la eficacia, ya que un recurso puede ser aceptable, pero escasamente eficaz $y$, a su vez, un recurso basado en técnicas y tratamientos empíricamente validados, pueden no ser eficaces porque no son aceptables para los usuarios.

\section{Referencias}

Andersson, A. y Titov, N. (2014). Advantages and limitations of Internet-based interventions for common mental disorders. World Psychiatry, 13, 4-11.

Andrews, G., Cuijpers, P., Craske, M.G., McEvoy, P. y Titov, N. (2010). Computer therapy for the anxiety and depressive disorders is effective, acceptable and practical health care: a meta-analysis. PLOS ONE, 5, e13196.

Antonacci, D. J., Bloch, R. M., Saeed, S. A., Yildirim, Y. y Talley, J. (2008). Empirical evidence on the use and effectiveness of telepsychiatry via videoconferencing: implications for forensic and correctional psychiatry. Behavioral Sciences \& the Law, 26(3), $253 \mathrm{e} 269$.

Baumeister, H., Reichler, L., Munzinger, M. y Lin, J. (2014).The impact of guidance on internet-based mental health interventions - a systematic review. Internet Interventions, 1(4), 205-215. Social Science \& Medicine, 71, 1308-1315.

Bee, P. E., Lovell, K., Lidbetter, N., Easton, K. y Gask, L. (2010). You can't get anything perfect: "User perspectives on the delivery of cognitive behavioural therapy by telephone". Social Science \& Medicine, 71, 1308-1315. ESEMed (2004). Disability and quality of life impact of mental disorders in Europe: Results from the European Study of the Epidemiology of Mental Disorders (ESEMeD) project. Acta Psychiatrica Scandinavica, 109, 38-46.

Froján-Parga, M. X., Galván-Domínguez, N., Iz- 
quierdo-Alfaro, I., Ruiz-Sancho, E. y Marchena-Giráldez, C. (2013). Análisis de las verbalizaciones desadaptativas del cliente y su relación con las verbalizaciones punitivas del terapeuta: un estudio de caso. Análisis y Modificación de Conducta, 39, 25-38.

Gun, S. Y., Titov, N. y Andrews, G. (2011). Acceptability of Internet treatment of anxiety and depression. Australas Psychriaty, 19, 259264.

Hommel, K. A., Hente, E., Herzer, M., Ingerski, L. M. y Denson, L. A. (2013). Telehealth behavioral treatment for medication nonadherence: a pilot and feasibility study. European Journal of Gastroenterology \& Hepatology, 25, 469-73.

Kessler, R., Petukhova, M., Sampson, A., Zaslavky, A. y Wittchen, H. (2012). Twelve month and lifetime prevalence and lifetime morbid risk of anxiety and mood disorders in the United States. International Journal of Methods in Psychiatric Research, 21(3), 169184.

Mitchell, J. E., Crosby, R. D., Wonderlich, S. A., Crow, S., Lancaster, K., Simonich, H., SwanKremeier, L., Lysne, C. y Myers, T. C. (2008). Behaviour Research and Therapy, 46, 581592.

Newman, M. G., Szkodny, L. E., Llera, S. J. y Przeworski, A. (2011). A review of technology assisted self-help and minimal contact therapies for anxiety and depression: Is human contact necessary for therapeutic efficacy?. Clinical Psychology Review, 31, 89-103. Peñate, W. (2012). About the effectiveness of telehealth procedures in psychological treatments. International Journal of Clinical and Health Psychology, 12, 475-487.

Peñate, W., Roca Sanchez, M. J., Pitti, C. T., Bethencourt, J. M., de la Fuente, J. A. y Gracia, R. (2014). Cognitive-behavioral treatment and antidepressants combined with virtual reality exposure for patients with chronic agoraphobia. International Journal of Clinical and Health Psychology, 14, 330-338.

Peñate, W., Roca-Sánchez, M. J. y Del PinoSedeño, T. (2014). Los nuevos desarrollos tecnológicos aplicados al tratamiento psicológico. Acta Colombiana de Psicología, 17,
91-101.

Pitti, C. T., Peñate, W., de la Fuente, J., Bethencourt, J. M., Roca-Sánchez, M. J., Acosta, L., Villaverde, M. L. y Gracia, R. (2015, en prensa). The combined use of virtual reality exposure in the treatment of agoraphobia. Actas Españolas de Psiquiatría.

Richards, D. y Richardson, T. (2012). Computerbased psychological treatments for depression: a systematic review and meta-analysis. Clinical Psychology Review, 32, 329-342.

Rush, B.y Scott, R. E. (2004). Approved Telehealth Outcome Indicator Guidelines: Quality, Access, Acceptability and Cost. Calgary Health Telematics Unit,University of Calgary.

Van Ballegooijen, W., Cuijpers, P., van Straten, A., Karyotaki, E. y Andersson, G. (2014). Adherence to Internet-Based and Face-to-Face Cognitive Behavioural Therapy for Depression: A Meta-Analysis. PLoS ONE, 9, e100674.

Wilson, G.T. (1996). Manual-based treatments: The clinical application of research findings. Behaviour Research and Therapy, 34, 295314.

Wilson, G. T. (1997). Treatment manuals in clinical practice. Behaviour Research and Therapy, 35, 205-210.

Wootton, B. M., Titov, N., Dear, B. F., Spence, J. y Kemp, A. (2011). The acceptability of Internet-based treatment and characteristics of an adult sample with obsessive compulsive disorder: an Internet survey. PLoS One, 6, e20548.

Yuen, E. K., Herbert, J. D., Forman, E. M., Goetter, E. M., Juarascio, A. S., Rabin, S., Goodwin, C. y Bouchard, S. (2013). Acceptance based behavior therapy for social anxiety disorder through videoconferencing. Journal of Anxiety Disorders, 27, 389-397. 


\section{ANEXO I}

\section{Cuestionario de aceptabilidad}

A continuación le pedimos que evalúe la el sitio web visitado. Son frases sencillas que se responden según el grado de acuerdo con el contenido de cada afirmación, marcando con un aspa (X) debajo de la opción elegida.

\begin{tabular}{|c|c|c|c|c|}
\hline Afirmaciones & $\begin{array}{l}\text { Muy de } \\
\text { acuerdo }\end{array}$ & De acuerdo & En desacuerdo & $\begin{array}{c}\text { Muy } \\
\text { en desacuerdo }\end{array}$ \\
\hline 1. Encontré la página web muy compleja & & & & \\
\hline $\begin{array}{l}\text { 2. Sé cómo conectarme como usuariom a } \\
\text { la web }\end{array}$ & & & & \\
\hline $\begin{array}{l}\text { 3. Entiendo cómo funciona la web de } \\
\text { manera global }\end{array}$ & & & & \\
\hline $\begin{array}{l}\text { 4. Considero que la mayoría de las iper- } \\
\text { sonas aprenderían muy rápidamente a } \\
\text { utilizar la web }\end{array}$ & & & & \\
\hline $\begin{array}{l}\text { 5. Encontré la web demasiado extensa al } \\
\text { recorrerla }\end{array}$ & & & & \\
\hline $\begin{array}{l}\text { 6. Me sentí muy confiado/a en el manejo } \\
\text { de la web }\end{array}$ & & & & \\
\hline $\begin{array}{l}\text { 7. Necesito aprender muchas más cosas } \\
\text { antes de manejarme bien en la web }\end{array}$ & & & & \\
\hline 8. Me ha gustado el diseño de la web & & & & \\
\hline 9. Me ha resultado fácil leer el contenido & & & & \\
\hline $\begin{array}{l}\text { 10. Recomendaría el uso de la web a perso- } \\
\text { nas que padecieran agorafobia }\end{array}$ & & & & \\
\hline
\end{tabular}

Finalmente, ¿Qué cosas cambiaría o mejoraría en la web?

\section{Agradecimientos}

Este estudio se ha realizado con el apoyo de los proyectos financiados PSI2009-09836 (Ministerio de Economía y competitividad), y PSI2013- 42912-R (Ministerio de Economía y Competitividad). 
